\title{
Expression profile and promoter analysis of HEPIS
}

\author{
FEN HU ${ }^{1}$ and YUNFENG ZHANG ${ }^{2}$ \\ ${ }^{1}$ Department of Biological Information, College of Life Sciences, North China University of Science and Technology, \\ Tangshan; ${ }^{2}$ Department of Life Sciences, Tangshan Normal University, Tangshan, Hebei 063000, P.R. China
}

Received February 13, 2017; Accepted August 1, 2017

DOI: $10.3892 /$ etm.2017.5374

\begin{abstract}
Human embryo lung cellular protein interacting with severe acute respiratory syndrome-coronavirus nonstructural protein-10 (HEPIS) is a novel transcriptional repressor, the expression profile and promoter activity of which have not been well studied. In the present study, in situ hybridization of RNA was used to study differential HEPIS expression levels in different types of cancer and normal tissues. A total of six truncated lengths of the HEPIS promoter regulatory sequences were cloned into the pGL3-basic vector, and reverse transcription-quantitative polymerase chain reaction (RT-qPCR) and dual luciferase reporter assays were performed. The results of RT-qPCR demonstrated that HEPIS expression levels differed across four breast cancer cell lines. The results of the dual luciferase reporter assays revealed that the activities of the reporter gene fragments spanning $-1334 /+373,-1203 /+373,-1060 /+373$ and $-899 /+373$ bp were higher compared with the reporter gene fragments spanning $-759 /+373$ and $-279 /+373$ bp. A search of the transcription factor database TRANSFAC identified numerous octamer transcription factor-1 (OCT-1), nuclear factor (NF) $\kappa \mathrm{B}$ and C-JUN transcription factor binding sites located on the HEPIS promoter (pHEPIS). Furthermore, the results revealed that mutations of the OCT-1 (-1236/-1223 bp), NF- $\kappa$ B (-1186/-1176 bp) and C-JUN (-856/-846 bp) sites on the human pHEPIS resulted in a decrease in luciferase activity. A chromatin immunoprecipitation assay revealed that OCT-1, NF- $\kappa \mathrm{B}$ and C-JUN bound to pHEPIS in a site-dependent manner at the basal state. The TRANSFAC database was used to analyze the pHEPIS of multiple species and several activator protein-1, NF- $\kappa \mathrm{B}$ and OCT-1 transcription factor binding sites were predicted. In conclusion, the results of the present study suggest that HEPIS is expressed at different levels in multiple organs and breast cancer cell lines. Furthermore,
\end{abstract}

Correspondence to: $\mathrm{Dr}$ Fen $\mathrm{Hu}$, Department of Biological Information, College of Life Sciences, North China University of Science and Technology, 57 Jianshenan Road, Tangshan, Hebei 063000, P.R. China

E-mail: ihufen@126.com

Key words: core promoter, expression profile, human embryo lung cellular protein interacting with severe acute respiratory syndrome-coronavirus nonstructural protein-10, transcriptional regulation these findings indicate that OCT-1, NF- $\kappa \mathrm{B}$ and C-JUN transcription factors are associated with transcriptional regulation of the HEPIS gene.

\section{Introduction}

The human embryo lung cellular protein interacting with severe acute respiratory syndrome-coronavirus nonstructural protein-10 (SARS-CoV nsp-10; HEPIS) gene is a novel gene that was initially discovered by Hong et al (1) in 2008 from a cDNA library of human embryo lung tissues. The HEPIS protein is able to interact with SARS-CoV nsp-10 (1). SARS-CoV nsp-10 is produced by the coronavirus main protease, which cleaves polyproteins ppla-pplab during infection; this protein is able to function as a viral transcriptase (2). The HEPIS protein consists of 147 amino acids and has several casein kinase II phosphorylation sites (1). In a previous study, HEPIS was demonstrated to interact specifically with the TATA sequence of the heat shock protein 70 promoter, suggesting that HEPIS may be associated with gene transcriptional regulation (1). However, the expression profile and promoter activity of HEPIS are yet to be elucidated.

Changes in the expression of specific gene products are regulated by a wide range of mechanisms, including transcriptional and translational regulation (3). Octamer transcription factor-1 (OCT-1), nuclear factor $\kappa \mathrm{B}(\mathrm{NF}-\kappa \mathrm{B})$ and activator protein 1 (AP-1) are important transcription factors that serve roles in cancer cell proliferation, survival, transformation, invasion, metastasis, angiogenesis and chemotherapy/radiotherapy resistance (4). OCTs are a class of transcription factor that bind to the 'ATTTGCAT' sequence of the gene promoter (5). OCT-1 (also termed POU2F1) is a ubiquitously expressed transcription factor containing a POU domain with a homeobox subdomain (6). OCT-1 serves an important regulatory role in cellular transcription via binding to a specific promoter octamer sequence on the target genes (7). Furthermore, OCT-1 binds to cofactors that interact with the POU DNA-binding domain to either positively or negatively regulate a variety of genes (8). Previous studies have reported that OCT-1 affects the occurrence and development of several cancers, including breast cancer (9), LNCaP prostate cancer (10), esophageal squamous cell carcinoma (11) and colorectal cancer (12). $\mathrm{NF}-\kappa \mathrm{B}$ is a dimeric transcription factor that belongs to the Rel/NF- $\kappa \mathrm{B}$ family and is formed by hetero- or homodimerization (13). $\mathrm{NF}-\kappa \mathrm{B}$ is known to serve a vital role in the regulation of inflammation, immunity, cell 
proliferation and apoptosis (13-16). AP-1, which is a dimeric transcriptional activator composed of Jun, Fos, activating transcription factor and musculoaponeurotic fibrosarcoma protein subunits $(17,18)$, serves important roles in the regulation of cellular proliferation, transformation, differentiation and apoptosis via binding to a common AP-1-binding site in the target gene promoter $(19,20)$.

In the present study, in situ RNA hybridization and reverse transcription-quantitative polymerase chain reaction (RT-qPCR) were used to detect the HEPIS gene expression profile in several organ tissues and breast cancer cell lines. The promoter activity of the HEPIS gene was also investigated. The first step was to identify the core HEPIS promoter to enable subsequent determination of the important transcription factors. The promoter region and transcription factor binding sites of the HEPIS gene were predicted by bioinformatics analysis. The AP-1, NF- $\kappa \mathrm{B}$ and OCT-1 binding sites of the HEPIS promoter region were identified using site-directed mutagenesis, dual luciferase reporter assays and chromatin immunoprecipitation (ChIP) assays, respectively.

\section{Materials and methods}

RNA in situ hybridization. A DNA microarray containing samples from 72 cases of tumor and normal tissue was obtained from Shaanxi Chaoying Biotechnology Co., Ltd. (cat. no. BCN721; Xian, China). The samples were from the following 12 organs: Esophagus, stomach, colon, rectum, liver, lung, kidney, breast, uterine cervix, ovary, prostate and pancreas; with 3 cores positive for cancer and 3 cores of adjacent normal tissue from each organ and one cancer tissue core and one adjacent normal tissue core per case. The following sense and antisense probes matching the HEPIS core responding sequence were used: Antisense, digoxigenin (DIG)-TCTGCCCATATGTCAGGATTGGAAATAATGGAT -3' and sense, DIG-ATCCATTATTTCCAATCCTGACAT ATGGGCAGA-3'. All probes were synthesized by Sangon Biotech Co., Ltd. (Shanghai, China). Hybridization procedures were performed as previously described (21). Staining was scored using a $0-3+$ scale. 0 , no staining; $1+, 2+$ and $3+$ indicate increased intensity of the staining. Sub-regions excluding necrosis, macrophages and infiltrated neutrophils and lymphocytes were selected and scored. The intensity score for an array spot is the mean of all its sub-regions.

Cell culture. MDA-MB-231, MCF-7, T-47D, ZR-75-30 and 293 T cells (China Center for Type Culture Collection, Wuhan, China) were maintained in high-glucose Dulbecco's modified Eagle's medium (Gibco; Thermo Fisher Scientific, Inc., Waltham, MA, USA) supplemented with $10 \%$ fetal bovine serum (Hyclone; GE Healthcare Life Sciences, Logan, UT, USA) and incubated at $37^{\circ} \mathrm{C}$ with $5 \% \mathrm{CO}_{2}$. $293 \mathrm{~T}$ cells were seeded at a density of $15 \times 10^{4}$ cells/well in 6 -well plates for quantitative ChIP assays. 293T cells were seeded at a density of $5 \times 10^{4}$ cells/well in 24-well plates for luciferase assays.

$R T-q P C R$. Total RNA was extracted from MDA-MB-231, MCF-7, T-47D and ZR-75-30 breast cancer cells using TRIzol reagent (Invitrogen; Thermo Fisher Scientific, Inc.), according to the manufacturer's protocol. The extracted total RNA $(0.5 \mu \mathrm{g}$ per sample) was then used to synthesize first-strand cDNA using a GoScript ${ }^{\mathrm{TM}}$ Reverse Transcription System kit (Promega Corporation, Madison, WI, USA), according to the manufacturer's protocol. The primers used for PCR were as follows: HEPIS, forward, 5'-ATGTGG CTCAGTTTGTCCTC-3' and reverse, 5'-AGCAAGATT TCCTCCAGGTC-3'; GAPDH, forward, 5'-TGACTTCAA CAGCGACACCCA-3' and reverse, 5'-CACCCTGTTGCT GTAGCCAAA-3'. GAPDH was used as an internal control. qPCR was performed using a SYBR Master Mixture (Takara Biotechnology Co., Ltd., Dalian, China) according to the manufacturer's protocol using the following cycling conditions: $95^{\circ} \mathrm{C}$ for $30 \mathrm{sec}$, followed by 40 cycles of $95^{\circ} \mathrm{C}$ for $5 \mathrm{sec}$ and $60^{\circ} \mathrm{C}$ for $30 \mathrm{sec}$. The expression of HEPIS was analyzed as previously described (22).

Plasmid construction. The promoter sequence of the HEPIS gene (pHEPIS) was obtained by PCR from MCF-7 cell genomic DNA using the following primers: pHEPIS-F1.7k (-1334), forward 5'-ATCCTCGAGCATCACAAGTAGGGC AGCAT-3'; pHEPIS-F1.6k (-1060), forward 5'-ATCCTCGAG GAGTCTTCAAAGGGAGTG-3'; pHEPIS-F1.4k (-1203), forward 5'-ATCCTCGAGTCCTGGTATGCCAAGAAA-3'; pHEPIS-F1.3k (-899), forward 5'-ATCCTCGAGCAAGCT GATAGCCACCAA-3'; pHEPIS-F1.1k (-759), forward 5'-ATC CTCGAGAGGTTGGCAGGCCGGATAT-3'; pHEPIS-F0.6k (-279), forward 5'-ATCCTCGAGCGAAGAGGAGGGAGG TAG-3'; pHEPIS-R (+373), reverse 5'-AGTAAGCTTACTTCG CACCTTCGGCTA-3'. PCR was performed using pyrobest DNA polymerase (Takara Biotechnology Co., Ltd.). The PCR amplification reaction system conditions and PCR products were purified as previously described (23). Purified products were cloned into the XhoI ( $\underline{\mathrm{CTCGAG}})$ and HindIII ( $\underline{\mathrm{AAGCTT}})$ restriction enzyme sites of the pGL3-basic vector (Promega Corporation) using T4 DNA ligase (Sangon Biotech Co., Ltd.) according to the manufacturer's protocol.

In the present study, the transcription factor database TRANSFAC (www.cbrc.jp/research/db/TFSEARCH.html) was used for the search, and several AP-1, NF- $\mathrm{B}$ and OCT-1 transcription factor-binding sites were predicted within the HEPIS promoter region. Site-directed mutageneses of theOCT-1 $(-1236 /-1223$, negative numbers indicate that it is upstream of the transcription initiation site), NF- $\kappa \mathrm{B}(-1186 /-1176)$ and C-JUN (-856/-846) binding sites in the HEPIS promoter were performed using a Quick Change Site-Directed Mutagenesis kit (Stratagene; Agilent Technologies, Inc., Santa Clara, CA, USA) according to manufacturer's protocol, using the following primers: pHEPIS-OCT-1-M, forward 5'-TTATAGGTGT CAAATTCATCATCACCATCAAAACTGCGTGCTTCTG CACTGAAACA-3' and reverse 5'-TGTTTCAGTGCAGAA GCACGCAGTTTTGATGGTGATGATGAATTTGACACC TATAA-3'; pHEPIS-NF- $\kappa$ B-M, forward 5'-GAGTCTTCA AAGGGAGTGGAATTACCTGGATCTTCTGTTG-3' and reverse 5'-CAACAGAAGATCCAGGTAATTCCAACTCCC TTTGAAGACTC-3'; pHEPIS-C-JUN-M, forward 5'-AAT AACAAATTCATCATTGTTAGTTTGTAGCAGGATTGC ACTGGAGACAGAGATTCC-3' and reverse 5'-GGAATC TCTGTCTCCAGTGCAATCCTGCTACAAACTAACAAT GATGAATTTGTTATT-3'. Underlined base pairs indicate mutation sites. 
Transfection and dual luciferase reporter assay. 293T cells were cotransfected with $1 \mu \mathrm{g}$ pGL3-basic vector, pHEPIS-1.7K, pHEPIS-1.6K, pHEPIS-1.4K, pHEPIS-1.3K, pHEPIS-1.1K, pHEPIS-0.6K, pHEPIS-1.7K-M-OCT-1, pHEPIS-1.6K-M-NF- $\mathrm{B}$, pHEPIS-1.3k-M-C-JUN or pHEPIS-1.7K-3M and $0.2 \mu \mathrm{g}$ pRL-TK (Promega Corporation) plasmid DNA/well in 24-well plates using Lipofectamine 2000 (Invitrogen; Thermo Fisher Scientific, Inc.) according to the manufacturer's protocol. The luciferase activity of the extracts was assessed $24 \mathrm{~h}$ following transfection using a Betascope analyzer Infinite M200, (Tecan Group Ltd., Männedorf, Switzerland) and analyzed as previously described (23). The pRL-TK plasmid containing the Renilla luciferase gene was used as an internal control.

ChIP assays. ChIP assays were performed according to the manufacturer's protocol using a Millipore ChIP assay kit (EMD Millipore, Billerica, MA, USA). The following primary antibodies were used: Rabbit polyclonal antibodies against $\mathrm{NF}-\kappa \mathrm{B}$ p65 (cat. no. ab7970, 1:200), OCT-1 (cat. no. ab66132, 1:200; both Abcam, Cambridge, UK) and C-JUN (cat. no. sc-1694, 1:100), and anti-rabbit normal immunoglobulin $\mathrm{G}$ (cat. no. sc-2345, 1:100; both Santa Cruz Biotechnology, Inc., Dallas, TX, USA) was used as an negative control. The above antibodies were used per chromatin sample and rotated overnight at $4^{\circ} \mathrm{C}$. Protein A/G Agarose/Salmon Sperm DNA Secondary antibody (1:400; cat. nos. 16-157 and 16-201; EMD Millipore) was added per sample for $1 \mathrm{~h}$ at $4^{\circ} \mathrm{C}$ with rotation. The amount of each specific DNA fragment in the immunoprecipitates was determined using PCR reactions with the following primers: OCT-1, forward 5'-ATGTAATCCAGTAGCCTGTC-3' and reverse 5'-CTCCCTTTGAAGACTCTGA-3'; NF- $\kappa$ B, forward 5'-TTCAGAGTCTTCAAAGGGAG-3' and reverse 5'-GCA TACCAGGAGACAATAAAC-3'; C-JUN, forward 5'-GCC ACCAACAATAACAAA-3' and reverse 5'-AGGAGGACA TTCACTTGC-3'. The PCR was performed using a PCR Master Mix (Sangon Biotech, Shanghai, China) according to the manufacturer's protocol using the following cycling conditions: $95^{\circ} \mathrm{C}$ for $30 \mathrm{sec}$, followed by 30 cycles of $95^{\circ} \mathrm{C}$ for $30 \mathrm{sec}$, $60^{\circ} \mathrm{C}$ for $30 \mathrm{sec}$ and $72^{\circ} \mathrm{C}$ for $10 \mathrm{sec} ; 72^{\circ} \mathrm{C}$ for $5 \mathrm{~min}$.

Statistical analysis. Statistical analysis was performed using SPSS 9.0 software (SPSS, Inc., Chicago, IL, USA). Data are presented as the mean \pm standard deviation. Student's t-test and one-way analysis of variance followed by a Dunnett's test were used to analyze data. $\mathrm{P}<0.05$ was considered to indicate a statistically significant difference.

\section{Results}

HEPIS expression profile in tissues and breast cancer cells. The HEPIS expression profile was detected by RNA in situ hybridization in a tissue microarray. HEPIS expression in esophageal squamous cell carcinoma and rectal adenocarcinoma tissues was the opposite of that in normal esophageal and rectal tissues (Table I; Fig. 1); HEPIS expression was positive in esophageal squamous cell carcinoma and negative in normal esophageal tissue, whereas it was positive in normal rectal tissue and negative in rectal adenocarcinoma. HEPIS was positively expressed in tumor and normal tissues

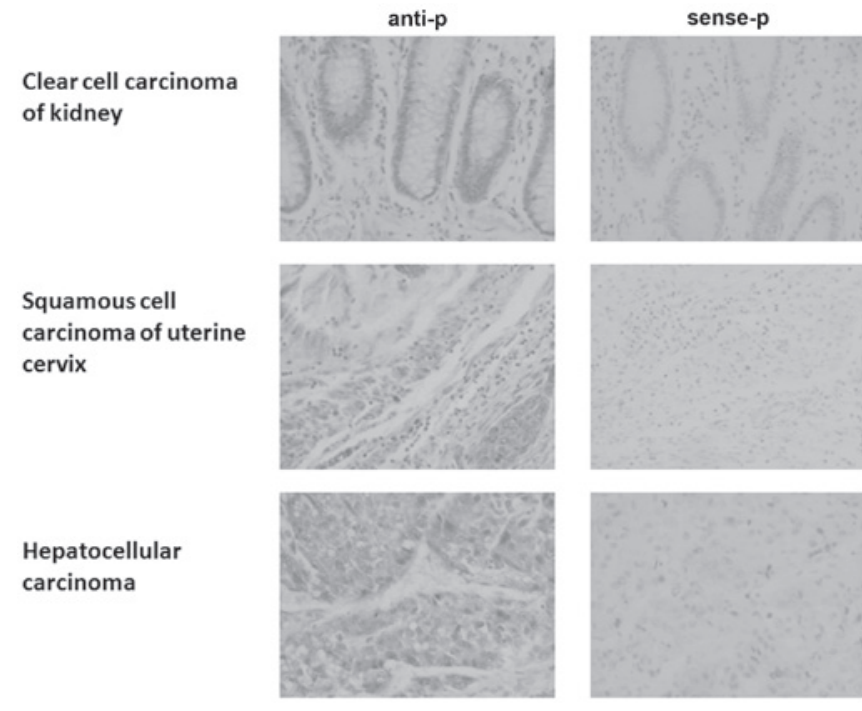

Figure 1. RNA in situ hybridization demonstrating HEPIS expression in clear cell carcinoma of the kidney, squamous cell carcinoma of the uterine cervix, and hepatocellular carcinoma. Magnification, x200. HEPIS, human embryo lung cellular protein interacting with severe acute respiratory syndrome-coronavirus nonstructural protein-10; anti-p, antisense probe used to detect HEPIS mRNA expression; sense-p, sense probe used as a negative control.

from the stomach, liver, colon, prostate, lung, uterine cervix and pancreas (Table I). The expression of HEPIS was positive in some tumor and normal tissues of the kidneys and ovaries and negative in others. HEPIS was positively expressed in nonspecific infiltrating duct carcinoma of the breast and partial positive expression was observed in normal breast tissue. HEPIS expression levels in four human breast cancer cell lines was examined using RT-qPCR (Fig. 2). The expression of HEPIS was significantly increased in the osteolytic breast cancer T-47D cell line compared with ZR-75-30, MDA-MB-231 and MCF-7 cells. HEPIS mRNA levels in T-47D cells were $\sim 8$-fold higher compared with MCF-7 cells $(\mathrm{P}<0.01)$, and in ZR-75-30, MDA-MB-231 cells were $\sim 1.8$-fold higher compared with MCF-7 cells $(\mathrm{P}<0.05)$. These results suggest that HEPIS is expressed at different levels in various organs and breast cancer cell lines.

Cloning and activity of the human HEPIS promoter. To understand the mechanism by which HEPIS gene transcripts are expressed, dual luciferase reporter assays were used to detect HEPIS promoter activity. A total of six different truncated lengths of the HEPIS promoter regulatory sequences were amplified and the PCR products were cloned into the pGL3-basic vector (Fig. 3A). Dual luciferase reporter assay analysis of the six recombined plasmids revealed that the $-1334 /+373,-1203 /+373,-1060 /+373$, and $-899 /+373$ bp reporter gene fragments exhibited higher activity levels compared with pGL3-basic $(\mathrm{P}<0.01)$; and the $-759 /+373$ bp and $-279 /+373$ bp reporter gene fragments exhibited higher activity levels compared with pGL3-basic $(\mathrm{P}<0.05$; Fig. 3B).

Mutations at transcription factor binding sites and luciferase activity analysis. To investigate whether these putative response elements regulate the transcription of HEPIS, 
Table I. HEPIS expression in multiple organ cancer and normal tissue.

\begin{tabular}{|c|c|c|c|c|}
\hline Organ & $\begin{array}{l}\text { Pathology } \\
\text { diagnosis }\end{array}$ & $\begin{array}{l}\text { Tissues/samples } \\
\text { (n) }\end{array}$ & $\begin{array}{l}\text { HEPIS mRNA-positive } \\
\text { tumors, } \mathrm{n}(+/++/+++)\end{array}$ & $\begin{array}{l}\text { HEPIS mRNA- } \\
\text { negative tumors, } \mathrm{n}\end{array}$ \\
\hline \multirow[t]{2}{*}{ Esophagus } & Squamous cell carcinoma & 3 & $3(0 / 3 / 0)$ & 0 \\
\hline & Normal tissue & 3 & 0 & 3 \\
\hline \multirow[t]{2}{*}{ Stomach } & Adenocarcinoma & 3 & $3(1 / 2 / 0)$ & 0 \\
\hline & Normal tissue & 3 & $3(0 / 1 / 2)$ & 0 \\
\hline \multirow[t]{2}{*}{ Colon } & Adenocarcinoma & 3 & $3(0 / 2 / 1)$ & 0 \\
\hline & Normal tissue & 3 & $3(0 / 0 / 3)$ & 0 \\
\hline \multirow[t]{2}{*}{ Rectum } & Adenocarcinoma & 3 & 0 & 3 \\
\hline & Normal tissue & 3 & $3(0 / 3 / 0)$ & 0 \\
\hline \multirow[t]{2}{*}{ Liver } & Hepatocellular carcinoma & 3 & $3(0 / 2 / 1)$ & 0 \\
\hline & Normal tissue & 3 & $3(0 / 0 / 3)$ & 0 \\
\hline \multirow[t]{2}{*}{ Lung } & Squamous cell carcinoma & 3 & $3(0 / 3 / 0)$ & 0 \\
\hline & Normal tissue & 3 & $3(3 / 0 / 0)$ & 0 \\
\hline \multirow[t]{2}{*}{ Kidney } & Clear cell carcinoma & 3 & $2(2 / 0 / 0)$ & 1 \\
\hline & Normal tissue & 3 & $1(1 / 0 / 0)$ & 2 \\
\hline \multirow[t]{2}{*}{ Breast } & Non-specific infiltrating duct carcinoma & 3 & $3(2 / 1 / 0)$ & 0 \\
\hline & Normal tissue & 3 & $2(1 / 1 / 0)$ & 1 \\
\hline \multirow[t]{2}{*}{ Uterine cervix } & Squamous cell carcinoma & 3 & $3(3 / 0 / 0)$ & 0 \\
\hline & Normal tissue & 3 & $3(3 / 0 / 0)$ & 0 \\
\hline \multirow[t]{2}{*}{ Ovary } & Serous cystadenocarcinoma & 3 & $1(1 / 0 / 0)$ & 2 \\
\hline & Normal tissue & 3 & $1(1 / 0 / 0)$ & 2 \\
\hline \multirow[t]{2}{*}{ Prostate } & Adenocarcinoma & 3 & $3(1 / 1 / 1)$ & 0 \\
\hline & Normal tissue & 3 & $3(2 / 1 / 0)$ & 0 \\
\hline \multirow[t]{2}{*}{ Pancreas } & Duct adenocarcinoma & 3 & $3(3 / 0 / 0)$ & 0 \\
\hline & Normal tissue & 3 & $3(0 / 3 / 0)$ & 0 \\
\hline
\end{tabular}

HEPIS, human embryo lung cellular protein interacting with severe acute respiratory syndrome-coronavirus nonstructural protein-10.

the OCT-1 (5'-CTATTTGCTTCTG-3', -1236/-1223 bp), NF- $\kappa$ B (5'-GGAATCCCCT-3', -1186/-1176bp), and C-JUN (5'-TTGAGTCAGG-3', -856/-846bp) response elements on the human HEPIS promoter were mutated to generate pHEPIS-1.7K-M-OCT-1, pHEPIS-1.6K-M-NF- $\kappa$ B and pHEPIS-1.3K-M-C-JUN, which were constructed individually (Fig. 4A). The dual luciferase assay results demonstrated that the luciferase activities of pHEPIS-1.7K-M-OCT-1, pHEPIS-1.6K-M-NF- $\kappa$ B and pHEPIS-1.3k-M-C-JUN were significantly decreased compared with the activities of pHEPIS-1.7K, pHEPIS-1.6K and pHEPIS-1.3K, respectively $(\mathrm{P}<0.05$; Fig. 4B), suggesting that C-JUN, OCT-1 and $\mathrm{NF}-\kappa \mathrm{B}$ activate the reporter. Furthermore, the OCT-1, NF- $\kappa \mathrm{B}$ and C-JUN binding elements of the HEPIS promoter were simultaneously mutated to generate pHEPIS-1.7K-3M. When all three sites were mutated, the pHEPIS-1.7K-3M promoter activity was significantly decreased compared with the pHEPIS-1.7K ( $\mathrm{P}<0.05$; Fig. 4B); however, the level of suppression with the three mutations did not exceed the combined level of suppression by the individual point mutations, which suggests that the three mutations act jointly. Taken together, these results suggest that the OCT-1, NF- $\mathrm{BB}$ and C-JUN sites serve an important role in inhibiting the transcriptional activity of HEPIS.
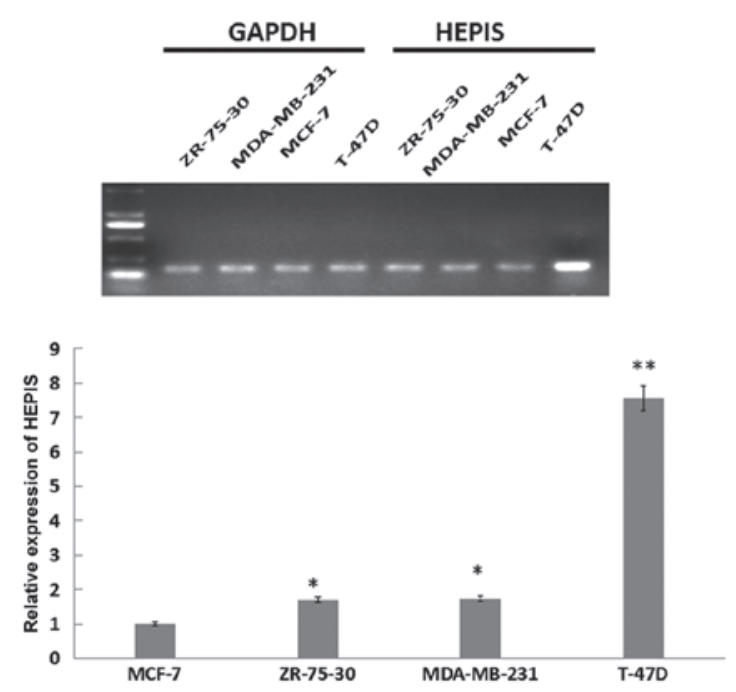

Figure 2. HEPIS mRNA levels in MDA-MB-231, MCF-7, T-47D and ZR-75-30 breast cancer cells. ${ }^{*} \mathrm{P}<0.05$ and ${ }^{* *} \mathrm{P}<0.01$ vs. MCF-7. HEPIS, human embryo lung cellular protein interacting with severe acute respiratory syndrome-coronavirus nonstructural protein-10.

Identification of transcription factors in the HEPIS promoter. Identifying the transcription factor binding sites within 
Table II. Analysis of HEPIS promoters in multiple species.

Transcriptional factor binding sites, $\mathrm{n}$

\begin{tabular}{|c|c|c|c|c|c|c|}
\hline Species & Context of HEPIS promoter (-2.0k) & OCT-1 & $\mathrm{NF}-\kappa \mathrm{B}$ & AP-1 & C-JUN & C-Fos \\
\hline Rattus norvegicus (Norway rat) & Chr3: 91195981-91197981 [-] & 11 & 0 & 6 & 2 & 0 \\
\hline Mus musculus (house mouse) & Chr2: 101629105-101631105 [-] & 13 & 11 & 6 & 2 & 1 \\
\hline Homo Sapiens (human) & Chr11: 36592229-36594229 [+] & 14 & 5 & 5 & 2 & 0 \\
\hline Bos Taurus (cattle) & Chr15: $67842229-67844229[+]$ & 17 & 3 & 9 & 4 & 1 \\
\hline Pan troglodytes (chimpanzee) & Chr11: $36583771-36585771[+]$ & 14 & 6 & 6 & 2 & 0 \\
\hline Canis lupus familiaris (dog) & Chr18: $31618122-31620122$ [-] & 15 & 3 & 7 & 4 & 3 \\
\hline Macaca mulatta (rhesus monkey) & Chr14: 29326253-29328253 [-] & 14 & 4 & 5 & 2 & 0 \\
\hline
\end{tabular}

HEPIS, human embryo lung cellular protein interacting with severe acute respiratory syndrome-coronavirus nonstructural protein-10; OCT-1, octamer-binding transcription factor 1 ; NF- $\kappa \mathrm{B}$, nuclear factor- $\mathrm{kB}$; AP-1, activator protein 1 .
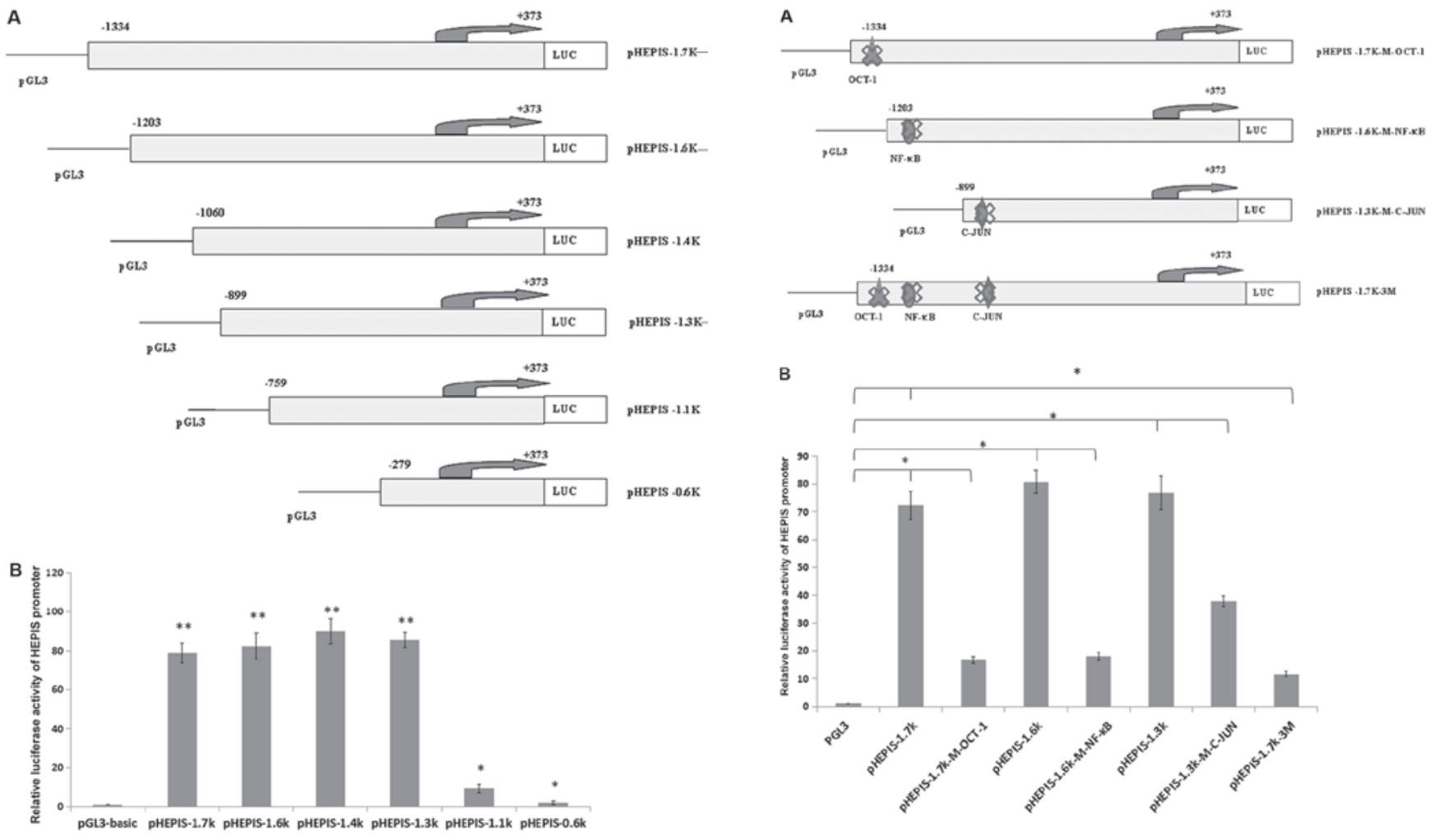

Figure 3. Cloning and activity of pHEPIS. (A) Six different truncated pHEPISs were cloned into a pGL3-basic LUC expression vector. These plasmids were designated as pHEPIS-1.7K, pHEPIS-1.6K, pHEPIS-1.4K, pHEPIS-1.3K, pHEPIS-1.1K and pHEPIS-0.6K. (B) Dual LUC activity assays of six pHEPIS constructs. Six recombinant vectors containing pHEPISs of different lengths and pRL-TK were cotransfected into $293 \mathrm{~T}$ cells. " $\mathrm{P}<0.05$, ${ }^{* *} \mathrm{P}<0.01$ vs. pGL3-basic. HEPIS, human embryo lung cellular protein interacting with severe acute respiratory syndrome-coronavirus nonstructural protein-10; pHEPIS, HEPIS promoter; LUC, luciferase.

the HEPIS promoter region is important for determining the mechanism of HEPIS gene transcription. To determine whether OCT-1, NF- $\mathrm{B}$ and C-JUN were able to bind to the endogenous HEPIS promoter, a ChIP assay was performed to investigate transcription factor binding. The results indicated

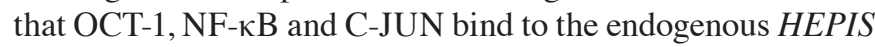

Figure 4. Mutation of transcription factor binding sites and LUC assay analysis of human pHEPIS. (A) The OCT-1, NF- $\kappa$ B and C-JUN elements were mutated individually on pHEPISs and designated as pHEPIS-1.7K-M-OCT-1, pHEPIS-1.6K-M-NF- $\kappa$ B and pHEPIS-1.3k-M-C-JUN. All three binding elements were mutated on the pHEPIS to generate pHEPIS-1.7K-3M. (B) Dual LUC activity assays of mutated pHEPIS constructs. A total of six recombinant vectors containing mutated pHEPIS fragments and pRL-TK were cotransfected into $293 \mathrm{~T}$ cells. ${ }^{*} \mathrm{P}<0.05$. LUC, luciferase; pHEPIS, human embryo lung cellular protein interacting with severe acute respiratory syndrome-coronavirus nonstructural protein-10 promoter; OCT-1, octamer-binding transcription factor $1 ; \mathrm{NF}-\kappa \mathrm{B}$, nuclear factor- $\kappa \mathrm{B}$.

promoter in $293 \mathrm{~T}$ cells, which suggests that they may serve an important role in regulating HEPIS expression (Fig. 5).

Analysis of HEPIS promoters in multiple species. Table II lists the putative HEPIS promoter among various species with 
DNA input (GAPDH primers)

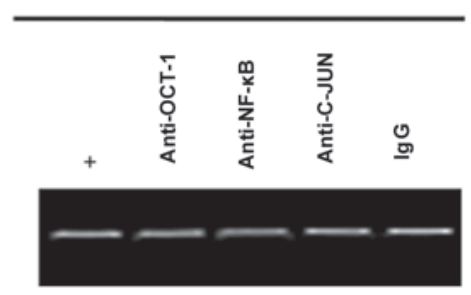

$\begin{array}{lll}\text { OCT-1 element } & \text { NF-KB element } & \text { C-JUN element } \\ \text { Spanning primers } & \text { Spanning primers } & \text { Spanning primers }\end{array}$

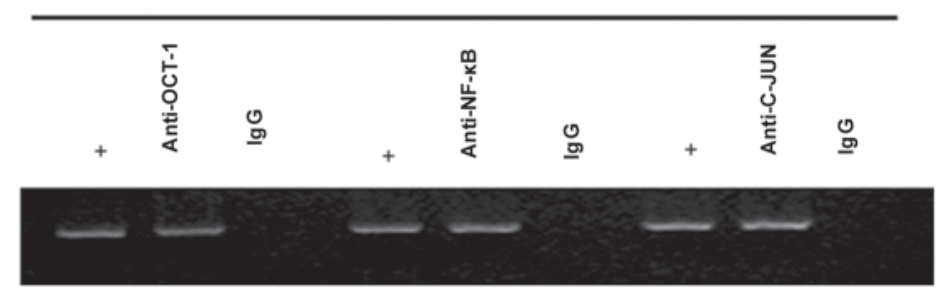

Figure 5. Binding of OCT-1, NF- $\mathrm{B}$ and C-JUN to the endogenous pHEPIS promoter was analyzed using a chromatin immunoprecipitation assay in $293 \mathrm{~T}$ cells. An amplified pHEPIS fragment with the OCT-1, NF- $\mathrm{B}$ and C-JUN elements is presented. The amount of DNA in the input confirms equal loading of chromatin. '+' indicates the positive control in which the template of genomic DNA fragments from 293T cells was used. OCT-1, octamer-binding transcription factor 1 ; NF- $\mathrm{B}$, nuclear factor- $\kappa \mathrm{B}$; pHEPIS, human embryo lung cellular protein interacting with severe acute respiratory syndrome-coronavirus nonstructural protein-10 promoter.

the same sequence length. The HEPIS promoter is conserved among vertebrates (Fig. 6). The sequence of the Homo sapiens (human) HEPIS promoter shares the highest homology (100\%) with that of Pan troglodytes (chimpanzees). The HEPIS promoters of Rattus norvegicus (Norway rats), Mus musculus (house mice), Bos taurus (cattle), chimpanzees, humans, Canis lupus familiaris (dogs) and Macaca mulatta (Rhesus monkeys) were analyzed using TRANSFAC and several AP-1, C-JUN, C-Fos, NF- $\kappa$ B and OCT-1 transcription factor binding sites were predicted within the promoter region (Table II).

\section{Discussion}

It has previously been reported that HEPIS is able to inhibit the proliferation of HeLa cells and may serve as an anti-oncoprotein (1). HEPIS is also able to inhibit the expression of the chloramphenicol acetyltransferase gene and may function as a factor of transcriptional repression (1). The aim of the present study was to determine the expression profile of the HEPIS gene and further elucidate the mechanism by which HEPIS transcriptional levels differ. RNA in situ hybridization (RISH) is a method of identifying the mRNA transcriptional expression pattern within the cytoplasm by hybridizing the sequence of interest to a labeled probe (24). Probes include radioactive probes and non-radioactive probes and RISH experiments performed with non-radioactive probes have several advantages over the radioactive procedures, including signal resolution, safety, shelf-life and cost (25). Due to the limited availability of the HEPIS antibody, the expression of the HEPIS gene in cancer and adjacent normal tissues in 12 organs was assessed using RNA in situ hybridization with a specific digoxigenin-labelled probe. However, due to the limited number of specimens available, it was necessary to further increase the number of specimens analyzed in order to obtain accurate results. HEPIS expression levels in four human breast cancer cell lines was examined using RT-qPCR, however, HEPIS expression in other types of cell lines remains unknown. Determining the differential expression of HEPIS allows analysis of its function in a variety of diseases.

Promoters control gene transcription. They may be located upstream of the gene transcription start site and can be very long (26). The binding of transcription factors to a promoter is an important mechanism by which gene expression is controlled (26). Investigating HEPIS promoter activity revealed

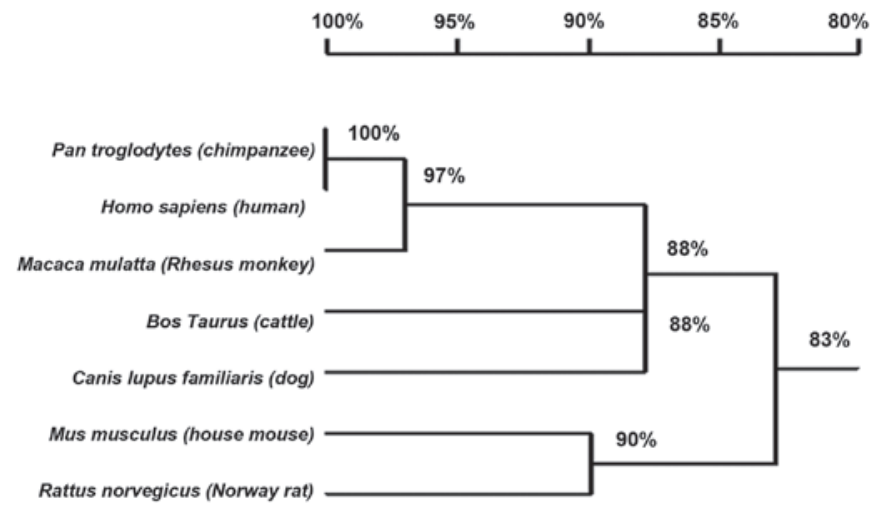

Figure 6. Homology tree of the HEPIS promoter $(2.0 \mathrm{~kb}$ upstream from the 5 '-end of the HEPIS gene). HEPIS, human embryo lung cellular protein interacting with severe acute respiratory syndrome-coronavirus nonstructural protein-10.

that the luciferase activity varied between pHEPIS-1.3k and pHEPIS-1.1k, suggesting that transcriptional regulation occurs at the-899/-759 bp region of the promoter. Furthermore, the results suggest that mutations of C-JUN (TTGAGTCAGG, -856/-846 bp), OCT-1 (CTATTTGCTTCTG, -1236/-1223 bp) and $\mathrm{NF}-\kappa \mathrm{B}$ (GGAATCCCCT, $-1186 /-1176 \mathrm{bp}$ ) result in a marked reduction in luciferase activity, which indicated that C-JUN, OCT-1 and NF- $\kappa \mathrm{B}$ are activators. However, no significant changes in luciferase activity were observed following truncation of the $-1334 / 1203 \mathrm{bp}$ and $-1203 /-1060$ bp regions. These results suggest that the $-1334 / 1203$ bp and -1203/-1060 bp regions also contain repressor-binding sites. The findings of the present study indicate that the apparent changes in transcriptional activity of the HEPIS gene may result from complex interactions of different transcription factors with the promoter. The association of HEPIS gene and the above transcription factors maybe widespread and therefore, further study is required.

In the present study, sequence analysis identified numerous transcription factor-binding sites within the HEPIS promoter sequence. Of these, OCT-1 NF- $\kappa \mathrm{B}$ and C-JUN were ubiquitously expressed; these have previously been reported to serve a variety of roles in the progression of numerous cancers $(5,9,16,17,19,20)$. The results of the ChIP assay indicated that OCT-1, NF- $\mathrm{BB}$ and C-JUN are able to bind to the endogenous HEPIS promoter in 293T cells. Several AP-1, 
C-JUN, C-Fos, NF- $\kappa$ B and OCT-1 transcription factor-binding sites were predicted within the putative HEPIS promoter in various species.

In conclusion, the results of this present research revealed that HEPIS has different expression levels in multiple types of cancer and normal tissues, and four breast cancer cell lines; and the OCT-1, NF- $\kappa \mathrm{B}$ and C-JUN transcription factors are associated with transcriptional regulation of the HEPIS gene. These findings provide further insight into the expression profile and the mechanism of HEPIS gene transcriptional regulation.

\section{Acknowledgements}

The present study was supported by the General Higher Education Young Talents Program of Hebei Province (grant no. BJ2014027) and the National Natural Science Foundation of China (grant no. 81302323).

\section{References}

1. Hong M, Li W, Wang L, Jiang L, Liu L, Zhao H and Li Q: Identification of a novel transcriptional repressor (HEPIS) that interacts with nsp-10 of SARS coronavirus. Viral immunol 21: 153-162, 2008.

2. Sawicki SG, Sawicki DL, Younker D, Meyer Y, Thiel V, Stokes H and Siddell SG: Functional and genetic analysis of coronavirus replicase-transcriptase proteins. PLoS Pathog 1: e39, 2005.

3. Edwards R, Machina A, McGregor G and van den Driessche P: A modelling framework for gene regulatory networks including transcription and translation. Bull Math Biol 77: 953-983, 2015.

4. Shanmugam MK, Nguyen AH, Kumar AP, Tan BK and Sethi G: Targeted inhibition of tumor proliferation, survival, and metastasis by pentacyclic triterpenoids: Potential role in prevention and therapy of cancer. Cancer Lett 320: 158-170, 2012.

5. Sterling K and Bresnick E: Oct-1 transcription factor is a negative regulator of rat CYP1A1 expression via an octamer sequence in its negative regulatory element. Mol Pharmacol 49: 329-337, 1996.

6. Lee MC, Toh LL, Yaw LP and Luo Y: Drosophila octamer elements and Pdm-1 dictate the coordinated transcription of core histone genes. J Biol Chem 285: 9041-9053, 2010.

7. Wang P, Wang Q, Sun J, Wu J, Li H, Zhang N, Huang Y, Su B, Li RK, Liu L, et al: POU homeodomain protein Oct-1 functions as a sensor for cyclic AMP. J Biol Chem 284: 26456-26465, 2009.

8. Malin S, Linderson Y, Almqvist J, Ernberg I, Tallone T and Pettersson S: DNA-dependent conversion of Oct-1 and Oct-2 into transcriptional repressors by Groucho/TLE. Nucleic Acids Res 33: 4618-4625, 2005.
9. Zhou C, Tong Y, Wawrowsky K, Bannykh S, Donangelo I and Melmed S: Oct-1 induces pituitary tumor transforming gene expression in endocrine tumors. Endocr-Relat Cancer 15: 817-831, 2008.

10. Obinata D, Takayama K, Urano T, Murata T, Kumagai J, Fujimura T, Ikeda K, Horie-Inoue K, Homma Y, Ouchi Y, et al: Oct1 regulates cell growth of LNCaP cells and is a prognostic factor for prostate cancer. Int J Cancer 130: 1021-1028, 2012.

11. Wang Z, Zhu S, Shen M, Liu J, Wang M, Li C, Wang Y, Deng A and Mei Q: STAT3 is involved in esophageal carcinogenesis through regulation of Oct-1. Carcinogenesis 34: 678-688, 2013.

12. Wang YP, Song GH, Chen J, Xiao C, Li C, Zhong L, Sun X, Wang ZW, Deng GL, Yu FD, et al: Elevated OCT1 participates in colon tumorigenesis and independently predicts poor prognoses of colorectal cancer patients. Tumour Biol 37: 3247-3255, 2016.

13. Karin $M$ and Ben-Neriah Y: Phosphorylation meets ubiquitination: The control of NF-[kappa] B activity. Annu Rev Immunol 18: 621-663, 2000.

14. Gebel HM, Braun DP, Tambur A, Frame D, Rana N and Dmowski WP: Spontaneous apoptosis of endometrial tissue is impaired in women with endometriosis. Fertil Steril 69: 1042-1047, 1998.

15. Huxford T, Malek S and Ghosh G: Structure and mechanism in NF-kappa B/I kappa B signaling. Cold Spring Harb Symp Quant Biol 64: 533-540, 1999.

16. Baldwin AS Jr: The NF-kappa B and I kappa B proteins: New discoveries and insights. Annu Rev Immunol 14: 649-683, 1996.

17. Shaulian E and Karin M: AP-1 in cell proliferation and survival. Oncogene 20: 2390-2400, 2001.

18. Eferl R and Wagner EF: AP-1: A double-edged sword in tumorigenesis. Nat Rev Cancer 3: 859-868, 2003.

19. Li GC, Gustafson-Brown C, Hanks SK, Nason K, Arbeit JM, Pogliano K, Wisdom RM and Johnson RS: c-Jun is essential for organization of the epidermal leading edge. Dev Cell 4: 865-877, 2003.

20. Shen G, Jeong WS, Hu R and Kong AN: Regulation of Nrf2, NF-kappaB, and AP-1 signaling pathways by chemopreventive agents. Antioxid Redox Signal 7: 1648-1663, 2005.

21. Hu F, Yang S, Lv S, Peng Y, Meng L, Gou L and Zhang X: Analysis of AC3-33 gene expression in multiple organ cancer and adjacent normal tissue by RNA in situ hybridization. Oncol Lett 9: 2795-2798, 2015.

22. Livak KJ and Schmittgen TD: Analysis of relative gene expression data using real-time quantitative PCR and the 2(-Delta Delta C(T)) method. Methods 25: 402-408, 2001.

23. Hu F, Meng Y, Gou L and Zhang X: Analysis of promoters and CREB/AP-1 binding sites of the human TMEM174 gene. Exp Ther Med 6: 1290-1294, 2013.

24. Denijn M, Schuurman HJ, Jacobse KC and De Weger RA: In situ hybridization: A valuable tool in diagnostic pathology. APMIS 100: 669-681, 1992.

25. Vale G and Dell'Orto P: Non-radioactive nucleic acid probes: Labeling and detection procedures. Liver 12: 243-251, 1992.

26. Farnham PJ: Insights from genomic profiling of transcription factors. Nat Rev Genet 10: 605-616, 2009. 